\title{
Kinetics and Spatial Distribution of Glial Derived Nerve Growth Factor during Experimental Peripheral Nerve Regeneration
}

\author{
Raquel Eguiluz-Ordóñez, Brenda Marquina-Castillo and Rogelio Hernández-Pando* \\ Sección de Patología Experimental, Departamento de Patología. Instituto Nacional de Ciencias Médicas y Nutrición "Salvador Zubiran", Mexico
}

*Corresponding author: Rogelio Hernández-Pando, Sección de Patología Experimental, Departamento de Patología. Instituto Nacional de Ciencias Médicas y Nutrición “Salvador Zubiran”, Mexico; Email: billing@researchopenworld.com

Received: August 11, 2021; Accepted: August 22, 2021; Published: August 27, 2021

\begin{abstract}
Nerve growth factors have been used therapeutically to enhance nerve regeneration with variable results, suggesting that before its use as therapeutic agents is important to determine their timing and spatial distribution during peripheral nerve regeneration. One of the most important growth factors in nerve regeneration is glial derived nerve factor (GDNF). Thus, in this study was determined the kinetics of gene expression and cellular sources of GDNF in Wistar rats after transection of the sciatic nerve and resection of $5 \mathrm{~mm}$ in its middle third.

Both proximal and distal nerve stumps were obtained at different time points with laser capture microdissection and used for RNA isolation for quantification of GDNF gene expression by Reverse Transcriptase Polymerase Chain Reaction. The cellular source was determined by immunohistochemistry. Our results showed that transcripts of GDNF were constantly expressed and exhibited two peaks, at $48 \mathrm{~h}$ being inflammatory macrophages the cells that showed the highest GDNF immunostaining. The second peak was seen at 17th to 26th days, being the Schwann cells from the proximal nerve stump the highest GDNF immunostained cells. Thus, GDNF is constantly produced at the site of the injury during peripheral nerve regeneration with two maximal time points, during early and late regeneration, suggesting that its local administration could be used therapeutically to increase nerve regeneration.
\end{abstract}

Keywords: Growth derived nerve factor, Nerve injury, Peripheral nerve regeneration, Laser microdisection

\section{Introduction}

Nerve growth factors are produced during embryo development, neurodegenerative diseases and after traumatic peripheral nerve injury [1]. During nerve regeneration diverse cells and its products participate, such as Schwann cells, macrophages and neurotrophic factors being one of the most important glial derived nerve factor (GDNF) [1].

Nerve growth factors have been administrated by various methods to enhance nerve regeneration [2]. These methods have had variable results in several experiments, perhaps because the timing and amount of the administrated factors have not been appropriate. Thus, it is important to know the kinetics of the neurotrophic factors production, in order to replicate the normal regeneration and in that way improve the reparative nerve process. The aim of the present study was to determine the kinetics and cellular sources of GDNF after section of the sciatic nerve in the rat.

\section{Materials and Methods}

Wistar rats six weeks old were anesthetized, the right sciatic nerve was exposed, dissected and transected in its middle third where $5 \mathrm{~mm}$ were resected. Group of three animals were euthanized at 6, 12, 24, 36 $\mathrm{h}$; and after $3,4,5,6,9,10,17,22,31,43$ days of nerve section. The surgical wound was opened, the sciatic nerve exposed and the distal part of the proximal stump was resected and tagged with silk suture in its proximal end. The same procedure was done in the proximal part of the distal nerve. Tissue samples were fixed and embedded in paraffin. Animals work was performed according to the guidelines of the Mexican Institutional Animal Care and the local committee (permit 264).

Specific areas of nerve regeneration were obtained by laser capture microdissection (LCM) using an XTTM Microdissection System Arcturus XT, isolating the distal portion of the proximal nerve and the most proximal part of the distal stump.

To determine the gene kinetics of GDNF, RNA was isolated from the nerve tissue fragments obtained by LCM. Reverse transcription was performed using $5 \mu \mathrm{g}$ RNA, oligo-dt and Omniscript kit (Qiagen, Inc). Real-time PCR was done using the 7500 RT-PCR system (Applied Biosystems, USA) and Quantitect SYBER Green Kit (Qiagen). Specific primers for GDNF transcripts were designed (Primer Express, Applied Biosystems, USA), $\beta$-actin was used as housekeeping gene. 
The same paraffin embedded tissue used for LMC was used for immunohistochemistry detection of GDNF, using a mouse monoclonal antibody (Santa Cruz Biotechnology, INC.) and antimouse rabbit immunodetector HRP/DAB (BIOSB, USA).

\section{Results}

Proximal and distal nerve stumps expressed GDNF transcripts in all the studied time points and showed two peaks, the highest was after

\section{GDNF}

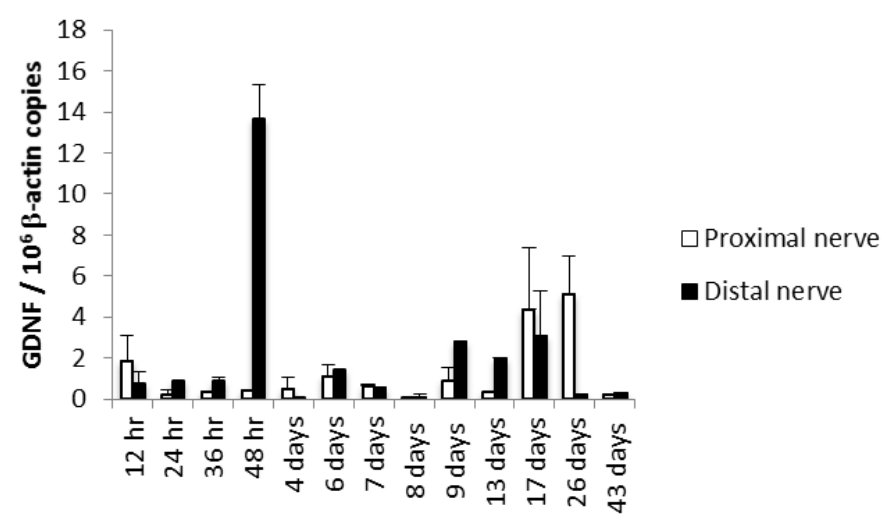

Figure 1: Kinetics of GDNF gene expression during peripheral nerve regeneration. Sciatic nerve was dissected, sectioned and a small fragment was removed in a large group of Wistar rats. Three rats were euthanized at the indicated time points and the injured sciatic nerve and surrounded tissues were dissected, fixed by immersion and embedded in paraffin. The proximal and distal nerve stumps were isolated from these paraffin blocks by laser microdisection and total RNA was purified and used to quantify GDNF gene expression by RT-PCR. The number of GDNF mRNA copies related to $10^{6} \mathrm{mRNA}$ copies of actin as housekeeping gene are shown. two days of nerve injury in the distal nerve ending, while the second peak was at 17 and 26 days in the proximal nerve stump (Figure 1).

Histologically, after $6 \mathrm{~h}$ there was acute inflammation with numerous mast cells around perineurial blood vessels and epineurium (Figure 2A). At $12 \mathrm{~h}$, the epineurium and perineurium showed connective tissue hyalinization, dilated blood vessels surrounded by numerous lymphocytes, monocytes and neutrophils (Figure 2B). The injured nerve showed vacuolization with cellular decrease. After two days, numerous lymphocytes and mainly macrophages were located around perineural vessels, on the epineurium and between nerve fascicles and muscle, the injured nerve showed detached Schwann cells. The epineurium show incipient granulation tissue, numerous mast cells, edema and focal rabdomyolisis (Figure 2C and 2D). Many macrophages showed strong GDNF immunostaining, while weak staining was seen in some fibroblasts, endothelial and Schwann cells (Figure 3A). At days 4 and 6, inflammation was mild with macrophages distributed between nerve fibers, in the epineurium and perineurium with some mast cells and fibroblasts. At day 7, the inflammatory infiltrate was slight and there were sprouts or nerve ramifications from the proximal nerve to the muscle tissue with some axons covered by Schwann cells (Figure 3E). The nerve sprouts showed irregular shape, some were nodules constituted by Schwann cells from day 17 to 26 (Figure 3F and 3G), surrounded by mild fibrosis and chronic inflammation that progressively decreased (Figure $3 \mathrm{H}$ ). Numerous new formed nerve sprouts were constituted by Schwann cells with strong GDNF immunostaining, being the highest in the principal sectioned nerve, while macrophages showed lesser immunoreactivity (Figure 3).
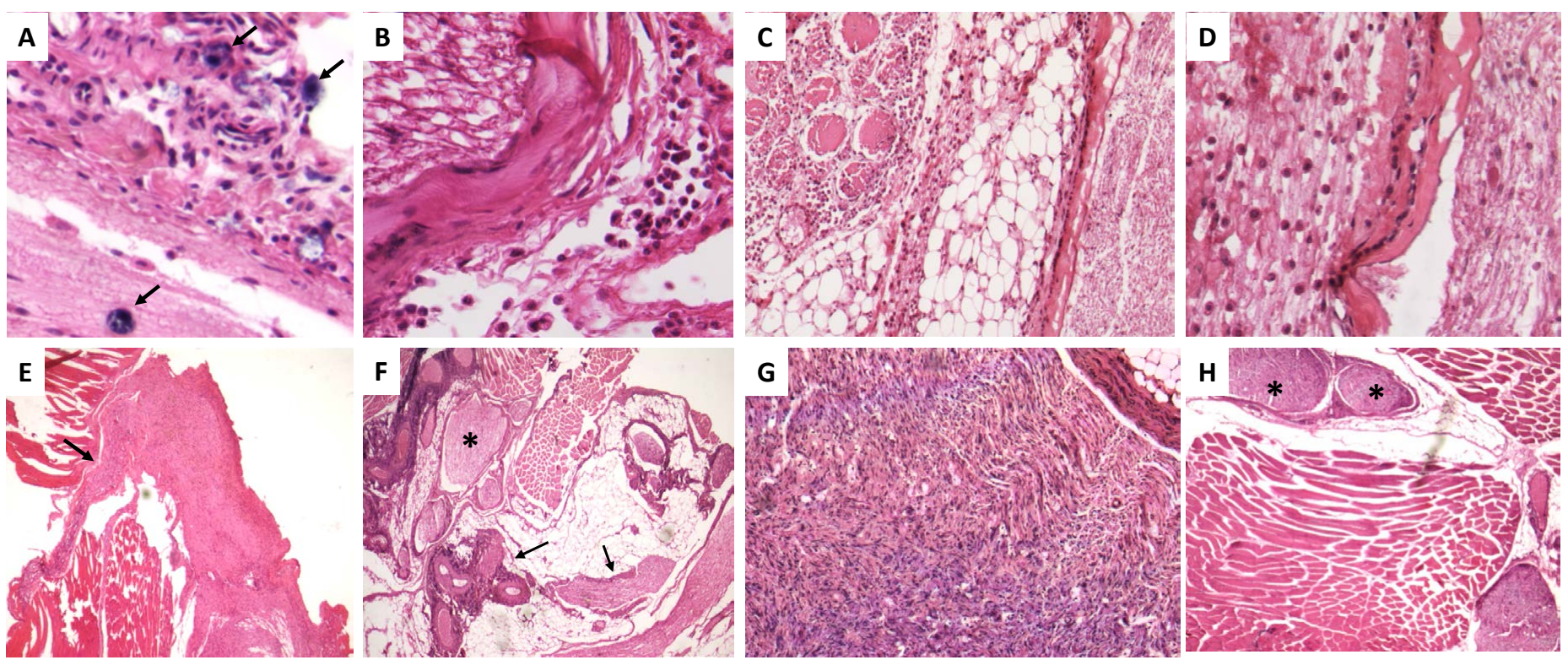

Figure 2: Representative micrographs of selected time points during peripheral nerve regeneration.

A) After $6 \mathrm{~h}$ of nerve injury there are acute inflammatory infiltrate in the epineurium with several mast cells (arrows).

B) Twelve hours after sciatic nerve section there are nerve vacuolization and hyalinization of epineural collagen (asterisk) with mild inflammatory infiltrate.

C) Two days after nerve injury there are intense inflammatory infiltrate into the nerve, its epineurium and neighbor adipose and muscular tissues.

D) High power micrograph of the lesion exhibited in $\mathrm{C}$ showed numerous macrophages dissecting the injured nerve, which showed extensive demielinization.

E) After one week of injury, there are slender prolongations or spouts (arrow) from the injured nerve.

F) After 17 days of injury there are numerous nerve sprouts with irregular shape and size (arrows), some of them have nodular morphology (asterisk).

G) High power micrograph of the same nerve showed in F exhibit numerous Schwann cells and few inflammatory cells.

H) After 43 days of nerve injury, some nerve sprouts show nodular organization resembling posttraumatic neuromas (asterisks). 

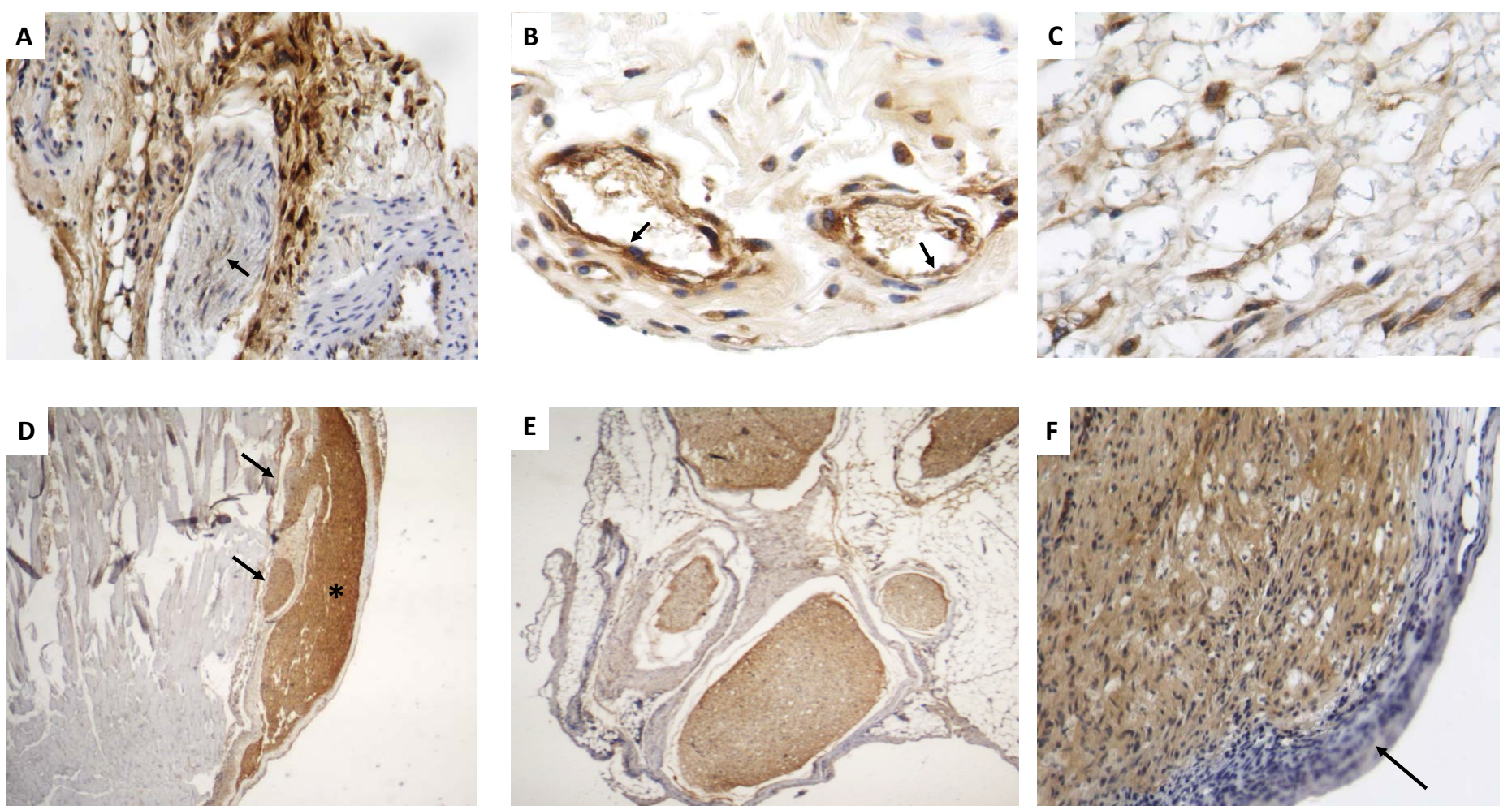

Figure 3: Representative micrographs of GDNF detection by immunohistochemistry during early and late sciatic nerve regeneration.

A) After $48 \mathrm{~h}$ of nerve injury there are numerous macrophages and fibroblasts that show strong GDNF immunostaining located in the perineurium, while few Schwann cells exhibit scarce immunereactivity (arrows).

B) Several perivascular inflammatory macrophages with strong GDNF immunostaining are seen around dilated blood vessels, which are revisted by strongly GDFN immunostained endothelium (arrows).

C) In the same early lesion, GDNF immunestained macrophages are seen into the injured nerve.

D) After 17 days of injury, regenerative sciatic nerve show strong GDNF immunostaining (asterisk), as well as its sprouts but in lesser intensity (arrows).

E) Collateral nerve ramifications or nerve sprouts are surrounded by thick fibrous epineurium and constituted by Schwann cells that show strong GDNF immunostaining.

F) High power micrograph show Schwann cells from regenerative nerve after 26 days of injury with strong GDNF immunostaining, while the inflammatory infiltrate is negative (arrow).

\section{Discussion}

The inflammatory response induced by peripheral nerve injury induces mast cells degranulation that release histamine and serotonin that enhance capillary permeability, facilitating macrophage migration [3]. Macrophages recruitment begins 2 to 3 days after nerve injury and peaks at about 7 or 14 days [4]. Macrophages and Schwann cells remove the injured tissue debris [5]. Macrophages secrete an enormous range of products, including cytokines and growth factors that are mitogenic for Schwann cells. Our results showed that GDNF is constantly produced and its highest gene expression was very early, after two days of nerve injury in the distal stump, being macrophages its most important cellular source as suggested by immunohistochemistry. Other lesser numerous GDNF immunostained cells were the endothelium and fibroblasts. Few Schwann cells showed slight GDNF immunoreactivity in this early regenerative response.

GDNF was originally identified in astrocytes and later in other cell types [6]. GDNF is a potent trophic factor for embryonic motoneurons that enhance their cholinergic maturation and reduce cell death after axotomy. GDNF overexpression by muscle cells highly increases the number of axons in neuromuscular junctions. Administration of GDNF results in motor unit enlargement and continuous synaptic remodeling at the neuromuscular junction [7]. GDNF is also produced by macrophages and activated microglia in response to striatal and spinal cord injury, where these cells remain at the wound site producing increasing amounts of GDNF. In experimental autoimmune neuritis, GDNF is produced by macrophages and $\mathrm{T}$ cells [8]. GDNF is up regulated after several types of peripheral nerve injury and its administration to adult rats can change the phenotype of nerve fibers from unmyelinated to myelinated, where Schwann cells also aid axonal outgrowth and remyelinate the regenerating axon [9]. Previous reports showed that $48 \mathrm{~h}$ after peripheral nerve injury GDNF expression increased and Schwann cells are responsible for its expression [10]. These observations are in agreement with our results of time course gene expression, but in disagreement with our immunohistochemistry results, which indicated that macrophages are the most important cellular source of GDNF after early nerve injury, while Schwann cells are apparently the source of the later second peak production. At this later time there are numerous nerve sprouts that showed strong GDNF immunostaining in Schwann cells. Thus, it seems that GDNF is related to the nerve sprouts production that apparently try to reconnect nerve endings, and this growth factor could also participate in the production of post-traumatic neuromas, because strong GDNF immunostaining was seen in nodular structures located near to the sectioned nerve. 


\section{References}

1. Cattin AL and Lloyd AC (2016) The multicellular complexity of peripheral nerve regeneration. Curr Opin Neurobiol 39:38-46. DOI:10.1016/j.conb.2016.04.005.

2. Zigmond RE and Echevarria FD (2019) Macrophage Biology in the Peripheral Nervous System after injury. Progress in Neurobiology 173:102-12. doi.org/10.1016/j. pneurobio.2018,12.001.

3. Zochodne DW (2000) The microenvironment of injured and regenerating peripheral nerves. Muscle Nerve Suppl 9: 33-38. [crossref]

4. Perry VH, Brown MC (1992) Role of macrophages in peripheral nerve degeneration and repair. Bioessays 14: 401-406. [crossref]

5. Stoll G, Griffin JW (1989) Wallerian degeneration in the peripheral nervous system participation of both Schwann cells and macrophages in myelin degradation. $J$ Neurocytol 18: 671-683. [crossref]

6. Zurn AD, Baetge EE, Hammang JP, Tan SA, Aebischer P (1994) Glial cell linederived neurotrophic factor (GDNF), a new neurotrophic factor for motoneurones. Neuroreport 30;6(1): 113-118. [crossref]
7. Keller-Peck CR, Feng G, Sanes JR, Yan Q, Lichtman JW, et al. (2001) Glial Cell LineDerived Neurotrophic Factor Administration in postnatal life results in motor unit enlargement and continuous synaptic remodeling at the neuromuscular junction. $J$ Neuroscience 21: 6136-6146. [crossref]

8. Ahn M, Jin JK, Moon C, Matsumoto Y, Koh C S, et al. (2010) Glial cell line-derived neurotrophic factor is expressed by inflammatory cells in the sciatic nerves of Lewis rats with experimental autoimmune neuritis. J Peripheral Nervous System 15: 104112. [crossref]

9. Höke A, Ho T, Craford TO, LeBel C, Hilt D, et al. (2003) Glial Cell Line-Derived Neurotrophic Factor alters axon Schwann Cell units and promotes myelination in unmyelinated nerve fibers. J Neuroscience 23: 561-567. [crossref]

10. Fadia NB, Bliley JM, DiBernardo GA et al. (2020) Long-gap peripheral nerve repair through sustained release of a neurotrophic factor in non human primates. Sci. Transl 12, eaav 7753. DOI: 10.1126/scitranslmed.aav.7753.

\section{Citation:}

Eguiluz-Ordóñez R, Marquina-Castillo B, Hernandez Pando R (2021) Kinetics and Spatial Distribution of Glial Derived Nerve Growth Factor during Experimental Peripheral Nerve Regeneration. Integr J Orthop Traumatol Volume 4(2): 1-4. 